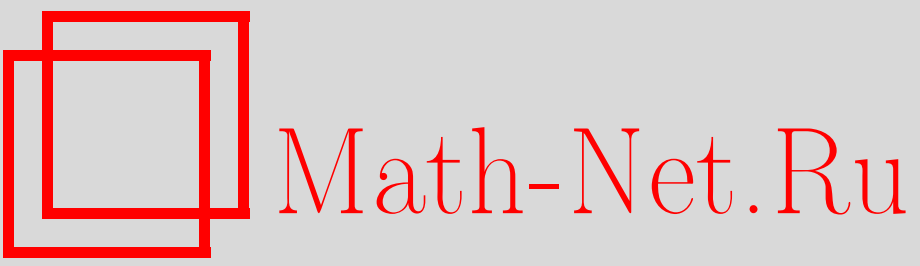

Д. П. Желобенко, С. М. Никольский, С. А. Теляковский, Бела Сёкефальви-Надь (некролог), УМН, 1999, том 54, выпуск 4, 143-146

DOI: https://doi.org/10.4213/rm198

Использование Общероссийского математического портала Math-Net.Ru подразумевает, что вы прочитали и согласны с пользовательским соглашением

http://www. mathnet.ru/rus/agreement

Параметры загрузки:

IP : 34.239 .49 .27

26 апреля 2023 г., 16:06:01 


\section{БЕЛА СЁКЕФАЛЬВИ-НАДЬ}

21 декабря 1998 года скончался выдающийся венгерский математик крупный специалист по классическому и функциональному анализу Бела Сёкеффальви-Надь.

Бела Сёкефальви-Надь родился 29 июля 1913 г. в городе Коложваре (њыне Клуж). В 1936 г. окончил университет в Сегеде, с 1948 г. - профессор Сегедского университета. В 1945 г. он был избран членом-корреспондентом Венгерской Академии наук, а в 1956 г. - академиком Венгерской АН. С 1953 г. являлся президентом Сегедского комитета ВАН.

Бела Сёкефальви-Надь имел систематические научные и дружеские контакты со многими советскими математиками, неоднократно посещал нашу страну. В 1971 г. он был избран иностранным членом Академии наук СССР. В 1980 г. ему (одновременно с академиком В.А. Котельниковым) была присуждена высшая награда нашей Академии - Золотая медаль им. М.В. Ломоносова. Благодаря своему высокому научному авторитету, он был избран также членом академий наук ряда других стран.

С 1975 г. Академия наук СССР и Венгерская Академия наук издают совместный журнал "Analysis Mathematica”. Б. Сёкефальви-Надь активно участвовал в его организации, а с начала выхода журнала был главным редактором венгерской секции редколлегии.

В течении нескольких десятилетий он возглавлял основанный А. Хааром и Ф. Риссом авторитетный журнал "Acta Scientiarum Mathematicarum", издаваемый Сегедским университетом.

В первые годы своего научного творчества Сёкефальви-Надь исследовал ряд важных экстремальных задач теории функций, в основном имеющих приложения в теории приближения функций.

В середине тридцатых годов X. Бор и Ж. Фавар нашли точные оценки максимума модуля периодических функций, имеющих ограниченную производную заданного порядка $r$ и ортогональных тригонометрическим полиномам порядка $m$. Эти результаты, которые можно рассматривать как двойственные к классическому неравенству Бернштейна для производных тригонометрических полиномов, позволили получить точное значение верхних граней наилучших приближений тригонометрическими полиномами на классах периодических функций с ограниченной производной порядка $r$.

Сёкефальви-Надь был одним из первых, кто откликнулся на эти исследования. В работах [1], [2] он обобщил неравенства Бора-Фавара на классы функций с коэффициентами Фурье $a_{k}, b_{k}$, для которых ряд

$$
\sum_{k=1}^{\infty} \lambda_{k}\left(a_{k}\left(\cos k x+\frac{r \pi}{2}\right)+b_{k}\left(\sin k x+\frac{r \pi}{2}\right)\right),
$$


где $\left\{\lambda_{k}\right\}$ - последовательность достаточно общего вида, является рядом Фурье функции, ограниченной по модулю единицей. Он получил и соответствующие оценки для наилучших приближений функций из этих классов. При этом он опирался на установленные им свойства нулей сумм рядов

$$
\sum_{k=m+1}^{\infty} \lambda_{k} \cos k x, \quad \sum_{k=m+1}^{\infty} \lambda_{k} \sin k x,
$$

последовательность коэффициентов которых трижды монотонна для ряда по косинусам и дважды монотонна для ряда по синусам (такие ряды называют ядрами Надя). Эти результаты вошли в литературу как "критерий Надя".

K этому кругу вопросов относятся и работы Сёкефальви-Надя [3], [4], в которых для многих классических методов суммирования тригонометрических рядов (средние Фейера, Чезаро, Рисса и других) найдены асимптотически точные оценки приближений на классах дифференцируемых функций.

Еще в начале века Э. Ландау и Ж. Адамар нашли точные оценки нормы в равномерной метрике первой производной функции через норму самой функции и норму ее второй производной. Интерес специалистов к задачам подобного рода возрос после того, как в 1939 г. А.Н. Колмогоров получил точную оценку нормы $f^{(k)}$ через нормы $f$ и $f^{(n)}$ при $0<k<n$, а затем выяснилась связь таких задач с рядом важных вопросов анализа. Б. Сёкефальви-Надю [5] принадлежит следуюший результат в этом направлении: он нашел точное значение множителя $K(p, q, r)$ в неравенстве

$$
\|f\|_{L_{q}} \leq K(p, q, r)\|f\|_{L_{r}}^{\alpha}\left\|f^{\prime}\right\|_{L_{p}}^{1-a}, \quad \alpha=(1-1 / p+1 / q) /(1-1 / p+1 / r),
$$

для функций, заданных на оси $(-\infty, \infty)$, у которых конечны все участвующие в нем величины, при естественных условиях $p \geq 1$ и $q>r>0$.

Этот результат до сих пор остается единственным случаем, когда задача Колмогорова точно решена для всех возможных значений параметрив $p, q$ и $r$.

Начиная с середины тридцатых годов, Сёкефальви-Надь активно занимается теорией линейных операторов в гильбертовом пространстве. Его ранний результат в этом направлении, опубликованный в 1936 г. [6] и навеянньй известной теоремой М. Стоуна, сводится к спектральному разложению однопараметрических (слабо непрерывных) групп эрмитовых операторов. Впоследствии этот результат был обобщен им на однопараметрические полугруппы нормальных опереторов [7], [8]. Его интересы в последующие годы связаны с исследованием предельного поведения спектра при аналитическом возмущении линейных операторов [8]-[10], с обобщениями теорем Пэли-Винера о полных биортогональных системах [11], с колебаниями неоднородных струн [12]. Отметим также изящный результат Сёкефальви-Надя, согласно которому всякая равномерно ограниченная однопараметрическая группа линейных операторов подобна унитарной однопараметрической группе.

С начала пятидесятых годов Сёкефальви-Надя особенно привлекает тематика, связанная с изучением проекций линейных операторов, т.е. операторов вида $A_{0}=\left.P_{0} A\right|_{H_{0}}$, где $A$ - линейньй оператор в гильбертовом пространстве $H, P_{0}$ - оператор ортогонального проектирования на подпространство $H_{0}$. Указанное соотношение записывается коротко в виде $A_{0}=\operatorname{pr} A$, причем оператор $A$ называется дилатацией оператора $A_{0}$ (или расширением оператора $A_{0}$ с выходом из пространства $H_{0}$ ). Пионерские работы в этом направлении принадлежат М. А. Наймарку, который показал, что изучение линейных операторов можно свести к изучению их дилатаций, обладающих более специальными свойствами. Например, каждый симметричный оператор обладает самосопряженной дилатацией (при надлежащем обобщении понятия дилатации на неограниченные операторы). Аналогично, каждый оператор сжатия $T(\|T\| \leq 1)$ обладает унитарной дилатацией: $T=\operatorname{pr} U$, где $U$ - унитарньй оператор. Сёкефальви-Надь показал, что последняя конструкция допускает следующую существенную модификацию: $T^{n}=\operatorname{pr} U^{n}$ для всех $n=1,2, \ldots$. Более того, аналогичный результат справедлив для более общих коммутативных семейств, состоящих из операторов сжатия [14], [15]. Все эти результаты имеют ряд интересных приложений, в том числе к эргодической теории линейных операторов [14]-[16]. 
Глубокие исследования по теории унитарных дилатаций проводились Сёкефальви-Надем частично в сотрудничестве с Ч. Фояшем. В основе этих исследований лежит понятие характеристической функции оператора сжатия $T$, определяемой посредством унитарной дилатации $U(T=\operatorname{pr} U)$ как некоторая операторнозначная аналитическая функция в единичном круге. Использование этого метода позволяет получить функциональную модель и построить функциональное исчисление (в классе Харди $H^{\infty}$ ) для операторов сжатия, по крайней мере, в том случае, когда вполне неунитарная часть оператора сжатия $T$ действует в сепарабельном гильбертовом пространстве. Проблема описания инвариантных подпространств оператора $T$ сводится к (также весьма нетривиальной) проблеме факторизации характеристической функции оператора $T$. Существенное продвижение удается получить для операторов "класса $C_{0}$ ", удовлетворяющих характеристическим уравнениям вида $u(T)=0$, где $0 \neq u \in H^{\infty}$.

Детальное изложение этой теории имеется в монографии Сёкефальви-Надя и Ч. Фояша [15] и в лекциях Сёкефальви-Надя [14], где приводится также подробная библиография. Часть дальнейших исследований Сёкефальви-Надя и его сотрудников посвящена приложениям общей теории к задачам математической физики, в том числе к теории рассеяния. Теория характеристических функций получила свое дальнейшее развитие в работах ряда математиков, в том числе в трудах одессой школы, возглавлявшейся М.Г. Крейном.

Работы Сёкефальви-Надя по теории линейных операторов существенно связаны с традициями венгерской школы функционального анализа, основанной $\Phi$. Риссом, одним из классиков функционального анализа. Публикация совместной монографии $\Phi$. Рисса и Сёкефальви-Надя [17], переведенной на многие языки и выдержавшей ряд изданий, явилась знаменательным событием, определившим по сушеству завершение классического этапа развития данной теории. Характерная особенность этой книги состоит в сочетании ясности изложения с детальным анализом большого фактического материала. До сих пор эта книга служит прекрасным пособием по изучению функционального анализа.

\section{Д. П. Желобенко, С. М. Никольский, С.А. Теляковский}

\section{СПИСОК ЛИТЕРАТУРЫ}

1. Über gewisse Extremalfragen bei transformierten trigonometrischen Entwicklungen. I. Periodischer Fall // Berichte der math.-phys Kl. Akad. der Wiss. zu Leipzig 1938. V. 90. P. 103-134

2. Über gewisse Extremalfragen bei transformierten trigonometrischen Entwicklungen. II. Nichtperiodischer Fall // Berichte der math.-phys Kl. Akad. der Wiss. zu Leipzig 1939. V. 91. P. 3-24

3. Approximation der Funktionen durch die arithmetischen Mittel ihrer Fourierschen Reihen // Acta Scint. Mathem. (Szeged). 1946. V. 11. P. 74-84

4. Sur une class générale de procéde de sommation pour les séries de Fourier // Hungarica Acta Mathem. 1948. V. 1. № 3. P. 14-52

5. Über Integralungleichungen zwischen einer Funktionen und ihrer Ableitung // Acta Sci. Math. (Szeged). 1941. V. 10. P. 64-74

6. Über messbare Darstellungen Liescher Gruppen // Math. Ann. 1936. V. 122. P. 286-296

7. On semi-groups of self-adjoint transformations in Hilbert space // Proc. Nat. Acad. Sci. USA. 1938. V. 24. P. 559-560

8. Spectraldarstellung linearer Transformationen des Hilbertschen Raumes. Berlin: Springer-Verlag, 1942

9. Perturbations des transformations autoadjointes dans l'espace de Hilbert // Comm. Math. Helv. 1946-1947. V. 19. P. 347-366

10. Perturbations des transformations lineares fermees // Acta Sci. Math. (Szeged). 1951. V. 14. P. $125-137$

11. Expansion theorems of Paley-Wiener type // Duke Math. J. 1947. V. 14. P. 975-978

12. Vibrations d'une corde non homogène // Bull. Soc. Math. France. 1947. V. 75. P. 193-208

13. On uniformly bounded linear transformations in Hilbert space // Acta Sci. Math. (Szeged). 1947. V. 11. P. $152-157$

14. Unitary dilations of Hilbert space operators and related topics. Providence: AMS Publ., 1974; Русский перевод: Добавление 2 к книге [17] 
15. Analyse harmonique des operateurs de l'espace di Hilbert. Budapest: Acad. Kiadó, 1967 (with Foias C.); Русский перевод: Гармонический анализ линейных операторов в гильбертовом пространстве. М.: Мир, 1970

16. Über Kontraktionen des Hilbertschen Raumes // Acta Sci. Math. (Szeged). 1943. V. 10. P. 202-205 (with Riesz F.)

17. Leçon d'analyse fonctionelle. Budapest: Acad. Kiadó, 1952 (with Riesz F.); Русский перевод: Лекции по функциональному анализу, 2-е изд. М.: Мир, 1979 